\title{
Paracoccidiodomicosis aguda/subaguda ganglionar con eosinofilia asociada
}

\section{Acute/subacute paracoccidioidomycosis with associated eosinophilia}

\author{
Juan Carlos Pineda, Ángela Patricia Montoya-Bernal, Herman Correa-López, \\ Xiomara Vanessa Cruz-Tangarife, María Cecilia Campiño-Martínez • Manizales (Colombia)
}

DOI: https://doi.org/10.36104/amc.2021.1921

\section{Resumen}

La paracoccidiodomicosis es una micosis endémica cuyo diagnóstico inicial puede ser difícil al compartir características clínicas de enfermedades granulomatosas y neoplásicas. Aunque su forma más frecuente es la crónica presentamos el caso de un paciente joven con un cuadro agudo/subagudo con linfadenopatías, eosinofilia periférica y una serología para hepatitis C falsamente positiva. El diagnóstico fue confirmado por histopatología y cultivo de tejidos y se dio manejo con anfotericina B ante el deterioro clínico por progresión del compromiso sistémico. (Acta Med Colomb 2021; 46. DOI: https://doi.org/10.36104/amc.2021.1921).

Palabras clave: paracoccidiodomicosis, paracoccidioides, linfadenopatía, eosinofilia, resultado del tratamiento, hepatitis $C$, pruebas serológicas.

\begin{abstract}
Paracoccidioidomycosis is an endemic mycosis whose initial diagnosis may be difficult, as it shares the clinical characteristics of granulomatous and neoplastic diseases. Although its chronic form is the most frequent, we present the case of a young patient with an acute/subacute presentation with lymphadenopathy, peripheral eosinophilia and a false-positive hepatitis $\mathrm{C}$ serology. The diagnosis was confirmed by histopathology and tissue culture, and he was treated with amphotericin B due to clinical deterioration secondary to progression of systemic involvement. (Acta Med Colomb 2021; 46. DOI: https://doi.org/10.36104/amc.2021.1921).
\end{abstract}

Key words: paracoccidioidomycosis, paracoccidioides, lymphadenopathy, eosinophilia, treatment outcome, hepatitis $C$, serologic tests.
Dr. Juan Carlos Pineda: Especialista en Medicina Interna, Hospital Departamental Universitario Santa Sofía de Caldas, Docente Facultad de Ciencias de la Salud, Universidad de Manizales; Dra. Ángela Patricia Montoya-Bernal: Residente en Medicina Crítica y Cuidado Intensivo, Universidad de Manizales; Dr Herman CorreaLópez: Especialista en Patología, Hospital Departamental Universitario Santa Sofía de Caldas; Xiomara Vanessa Cruz-Tangarife y María Cecilia Campiño-Martínez: Bacteriólogas Hospital Departamental Universitario Santa Sofía de Caldas. Manizales (Colombia).

Correspondencia: Dr. Juan Carlos Pineda. Manizales (Colombia).

E-mail: jpinedar@umanizales.edu.co Recibido: 18/VI/2020 Aceptado: 7/VII/2020

\section{Introducción}

La paracoccidiodomicosis (PCM) es una micosis sistémica endémica de América Latina producida por Paracoccidioides brasiliensis, es más frecuente en Brasil que tiene $80 \%$ de los casos y le siguen Colombia, Ecuador, Venezuela, Bolivia y Argentina. La incidencia en nuestro país es de 0.5-2.2/100 000 habitantes/año, siendo Caldas uno de los departamentos más afectados con una tasa de 2.9/1 000000 habitantes/año. El sexo masculino y la agricultura, sobre todo la exposición a cultivos de café y tabaco, han sido reconocidos como factores de riesgo para contraer el hongo, mientras el tabaquismo, la desnutrición y el alcoholismo están asociados con el desarrollo de la enfermedad sintomática $(1,2)$.

El germen se adquiere por vía respiratoria y compromete predominantemente pulmones, ganglios linfáticos, muco- sas, piel, glándulas adrenales y hueso. Teniendo esto en cuenta se han descrito cuatro estados clínicos: la forma aguda/subaguda o juvenil que afecta a los menores de 30 años y se desarrolla en pocas semanas dando cuenta de 10-15\% de los casos, la crónica o del adulto que se observa entre los 30 y 50 años y tarda meses en progresar correspondiendo a $80-90 \%$ de los casos, la subclínica o asintomática y la residual o secuelar $(3,4)$. Presentamos el caso de un paciente con una forma aguda/subaguda con compromiso ganglionar y eosinofilia.

\section{Presentación del caso}

En un hospital de alta complejidad del departamento de Caldas, en la zona cafetera de Colombia, fue atendido en agosto de 2019 un hombre de 25 años con un mes de 
dolor mesogástrico, fiebre, pérdida de $6 \mathrm{~kg}$ de peso y diaforesis. No tenía antecedentes importantes excepto que anteriormente trabajó como agricultor, consumía cigarrillo y cannabinoides. El examen físico reveló un índice de masa corporal de 19.4, adenopatías cervicales y axilares, sin ningún otro hallazgo patológico. En los exámenes de laboratorio había un hemograma con 16400 leucocitos/ $\mu \mathrm{L}, 4000$ eosinófilos $/ \mu \mathrm{L}, 8800$ neutrófilos $/ \mu \mathrm{L}, 2300$ linfocitos $/ \mu \mathrm{L}$, hemoglobina $13.5 \mathrm{~g} / \mathrm{dL}$ y 565000 plaquetas/ $\mu \mathrm{L}$; proteína C reactiva $25 \mathrm{mg} / \mathrm{dL}$ (rango normal $<1 \mathrm{mg}$ ) dL) y albúmina $3.3 \mathrm{~g} / \mathrm{dL}$; el extendido de sangre periférica, deshidrogenasa láctica, pruebas de función renal, hepática y de coagulación eran normales. La tomografía abdominal mostró ganglios linfáticos retroperitoneales aumentados de tamaño (Figura 1A) y la tomografía del tórax al igual que la espirometría eran normales. La endoscopia de vías digestivas altas encontró esofagitis aguda y erosiva. Se realizaron serologías para VIH y hepatitis B que fueron negativas, mientras que la de hepatitis $\mathrm{C}$ fue positiva con una carga viral negativa. Se tomó una biopsia de ganglio cervical que reportó necrosis de caseificación, tinción negativa de Ziehl-Neelsen y tinciones de hongos positivas para paracoccidioides spp (Figura 2), luego se realizó una inmunodifusión en suero para hongos que fue reactiva para paracoccidioidina. La electroforesis de proteínas en suero evidenció pico monoclonal en región gamma, $\mathrm{IgE}>$ $2.000 \mathrm{UI} / \mu \mathrm{L}$ (rango normal $<150 \mathrm{UI} / \mu \mathrm{L}$ ), $\operatorname{IgG} 3153 \mathrm{mg} / \mathrm{dL}$ (rango normal 600-1808 mg/dL) e IgM $395 \mathrm{mg} / \mathrm{dL}$ (rango normal 50-283 mg/dL), mientras la IgA fue normal. Con estos hallazgos se consideró una PCM aguda/subaguda leve y se dio manejo intrahospitalario con itraconazol oral 200 $\mathrm{mg}$ c/8h por cinco días y luego egresó con prescripción de $200 \mathrm{mg} /$ día hasta completar nueve meses.
Sin embargo, el paciente abandonó el manejo y seguimiento ambulatorio y regresó a urgencias cinco meses después por la misma sintomatología, tenía aumento del tamaño de las adenopatías con respecto a la valoración previa, ictericia en escleras y signos clínicos de ascitis. En ese momento los laboratorios mostraban 20000 leucocitos $/ \mu \mathrm{L}, 2200$ eosinófilos $/ \mu \mathrm{L}, 15400$ neutrófilos $/ \mu \mathrm{L}, 1400$ linfocitos $/ \mu \mathrm{L}$, hemoglobina $8.3 \mathrm{~g} / \mathrm{dL}$, hematocrito $25.6 \%$, volumen corpuscular medio $82.7 \mathrm{fl}, 274000$ plaquetas $/ \mu \mathrm{L}$, bilirrubina total $2.5 \mathrm{mg} / \mathrm{dL}$, bilirrubina directa $1.8 \mathrm{mg} / \mathrm{dL}$, bilirrubina indirecta $0.7 \mathrm{mg} / \mathrm{dL}$, fosfatasa alcalina $613 \mathrm{UI} / \mathrm{l}$ (rango normal 38-126 UI/ $\mu \mathrm{L}$ ), gammaglutamil transferasa $1671 \mathrm{UI} / 1$ (rango normal $15-73 \mathrm{UI} / \mu \mathrm{L}$ ) con transaminasas normales. Una nueva tomografía de abdomen evidenció aumento de las adenopatías retroperitoneales y hepatoesplenomegalia (Figura 1B) y en la de tórax aparecieron adenopatías mediastinales. Se repitió la biopsia de ganglio cervical con hallazgos histopatológicos similares a la previa, tinciones y cultivos de tejido que descartaron sobreinfección por bacterias y mycobacterias y un cultivo para hongos con medio Sabouraud y agar papa-dextrosa reveló la presencia de formas cerebriformes blancas con presencia del hongo (Figura 3). Durante su hospitalización tuvo dolor abdominal de muy difícil control y la ascitis aumentó por lo cual se llevó a paracentesis, los estudios de líquido peritoneal mostraron 1200 linfocitos $/ \mu \mathrm{L}$, sin otras células, tinciones y cultivos para gérmenes usuales negativos, reacción en cadena de polimerasa (PCR) para mycobacterium tuberculosis negativa y citología negativa para neoplasia, el gradiente albúmina sérica/líquido ascítico era 1.2 con dúplex de vasos portales negativo para trombosis e hipertensión portal. Se decidió ante la progresión de su compromiso sistémico administrar anfotericina B intravenosa por tres semanas, alcanzando

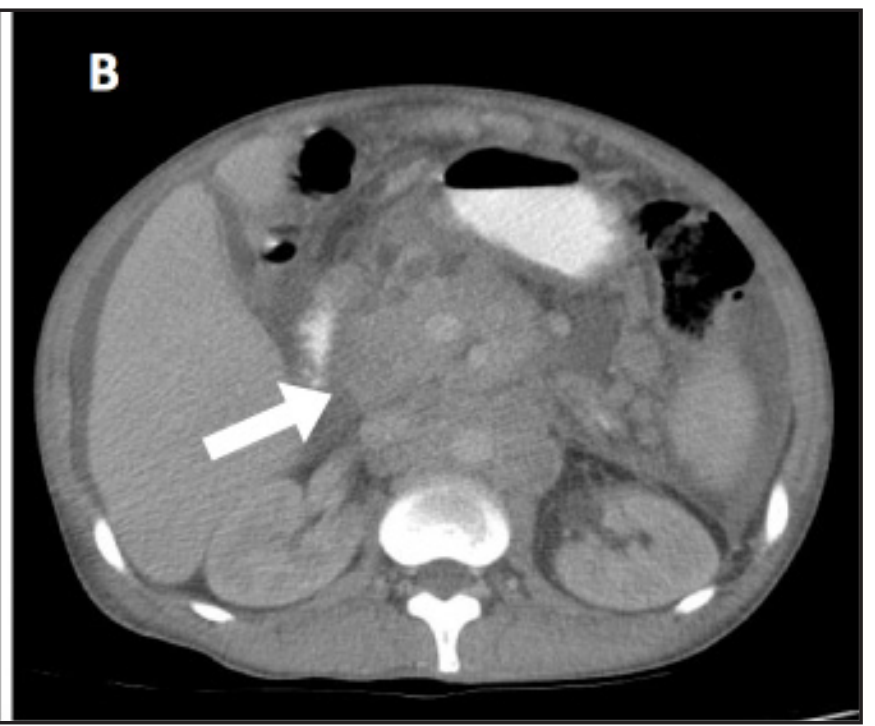

Figura 1. Tomografía computarizada contrastada de abdomen. A. Imagen del inicio del cuadro: múltiples adenopatías con áreas de necrosis central y refuerzo poscontraste en la transcavidad de los epiplones, hilio hepático, para-aórticas y en mesenterio (ver flecha). B. Imagen luego de 5 meses de abandono del tratamiento: grandes conglomerados de adenopatías retroperitoneales con aumento de tamaño y número respecto al estudio previo (ver flecha). 


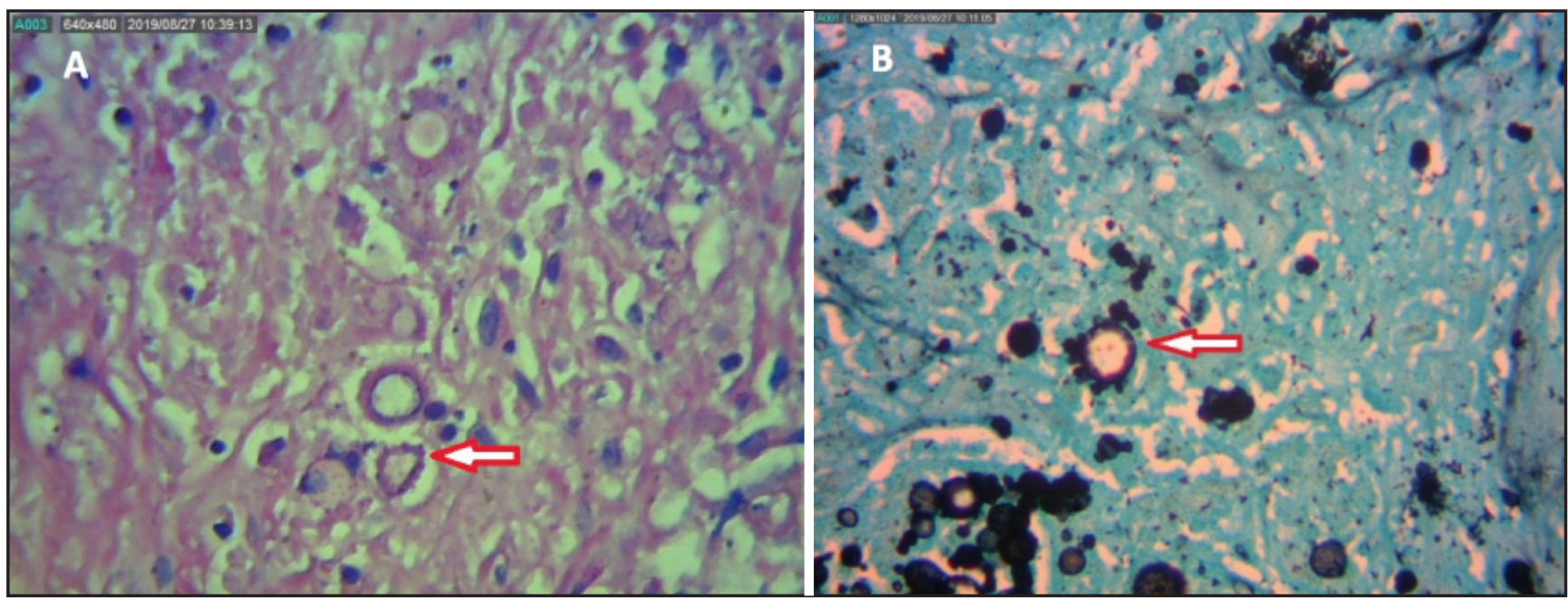

Figura 2. Histopatología de ganglio cervical. A. Tinción de ácido peryódico de Schiff con magnificación 40X. B. Tinción de Gomori-Grocott con magnificación 40X. Numerosas formaciones esferoidales u ovoides entre 10 y 15 micras de diámetro las cuales presentan remarcamiento capsular con gemaciones en "timón de barco" (ver flechas).

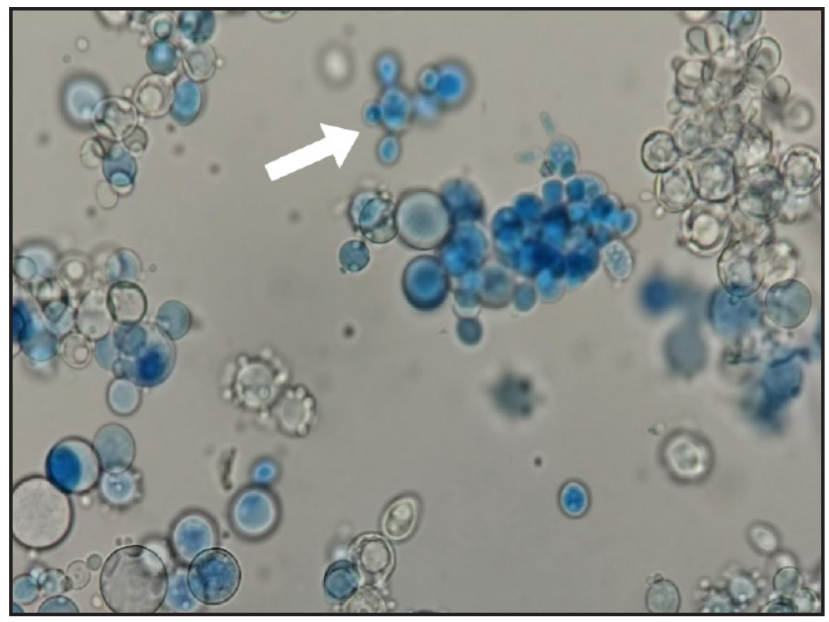

Figura 3. Cultivo de ganglio linfático. Tinción de azul de lactofenol con magnificación 40x. Evidencia de gemaciones en "timón de barco" (ver flecha).

1050 mg de dosis acumulada, luego se indicó manejo ambulatorio con trimetoprim-sulfametoxazol (TMP-SMX) oral $960 \mathrm{mg} \mathrm{c} / 12 \mathrm{~h}$ por un mes luego $480 \mathrm{mg} \mathrm{c} / 24 \mathrm{~h}$ por dos años, sin embargo, el paciente nuevamente abandonó el seguimiento y se desconoce su estado actual.

\section{Discusión}

La PCM ofrece un reto diagnóstico, debido a que sus manifestaciones clínicas, especialmente en la forma aguda/ subaguda, asemejan otras afecciones como el linfoma Hodgkin y la tuberculosis $(4,5)$. En este paciente la sospecha inicial fue un trastorno linfoproliferativo. La afectación pulmonar es el hallazgo más frecuente encontrándose en $64 \%$ de los casos, en la mucosa oral en $50 \%$, mientras que las linfadenopatías generalizadas corresponden apenas a $28 \%$ (6). Esto depende del tipo de presentación clínica, siendo la forma crónica la más frecuente y es donde se observa el compromiso del pulmón con hallazgos radiológicos de opacidades en vidrio esmerilado y nódulos cavitados, tal como se ha descrito en reportes de caso de nuestro medio en pacientes con formas crónicas leves y otro más severo con crisis adrenal y paro cardiorrespiratorio asociados (7, 8). La forma aguda/subaguda se observa en pacientes jóvenes y tiende a involucrar más los ganglios linfáticos, debido a la diseminación hematógena del hongo facilitada por la inmadurez del sistema inmune (6). En un estudio de cohorte de pacientes pediátricos encontraron $87 \%$ con linfadenopatías generalizadas, $75 \%$ fiebre y $65 \%$ pérdida de peso, pero sólo 5\% tenían dolor abdominal y $3 \%$ ascitis (9). En nuestro medio hasta ahora sólo están descritos dos casos de PCM aguda/subaguda, siendo uno de ellos en una paciente de 12 años, lo que contrasta con nuestro paciente quien ya estaba en la tercera década de la vida, tenía dolor abdominal marcado y ascitis $(10,11)$.

En los hallazgos de laboratorio de la PCM aguda/subaguda hay presencia de anemia en $88 \%$ de los casos, leucocitosis en $78 \%$, hipoalbuminemia en $65 \%$, hipergammaglobulinemia en $81 \%$ y además eosinofilia de $30-78 \%$ de los casos $(4,9)$, tal como se encontró en nuestro paciente. El aumento de los eosinófilos es un hallazgo muy llamativo que se ha descrito en otro reporte de caso de nuestro país y se atribuye a altos niveles de IL-5 y una respuesta celular Th2, aunque a diferencia de nuestro paciente no hubo compromiso hepático ni ascitis (11). La marcada producción de inmunoglobulinas puede explicar el falso positivo de los anticuerpos contra hepatitis $\mathrm{C}$, lo cual ha sido descrito en un paciente en diálisis peritoneal con peritonitis por Candida parapsilosis (12); sin embargo, a la fecha es el primer reporte de este hallazgo en personas con PCM. Si bien los cultivos y la histopatología permiten reconocer la presencia del hongo, el tiempo que se tarda en obtener los resultados puede retrasar la terapia, por lo que el estudio serológico de anticuerpos podría ayudar a un diagnóstico más precoz, aunque tiene el inconveniente de la reactividad cruzada con otras micosis y alta variabilidad 
entre los resultados de diferentes laboratorios $(5,13)$. En Colombia se ha estudiado la PCR anidada para detectar el gen que codifica la proteína de membrana gp43 del paracoccidioides en muestras de tejido, lavado broncoalveolar y esputo, encontrando una sensibilidad y especificidad de $100 \%$, por lo que podría ser otra herramienta diagnóstica más precisa y rápida (14).

El manejo dado a nuestro paciente se hizo de acuerdo con las recomendaciones de las guías más recientemente publicadas (4). Se usó itraconazol como primera línea debido a que al principio tenía un cuadro leve, este medicamento ofrece una curación más temprana cuando se compara con TMP-SMX, aunque algunos autores establecen que también es más eficaz $(15,16)$. Sin embargo, nos enfrentamos a la dificultad en la adherencia que generó progresión de la enfermedad con aparición de un cuadro más severo que requirió anfotericina $\mathrm{B}$ con la cual se han descrito tasas de curación de $100 \%$, luego de un año de seguimiento (17). En el manejo crónico el TMP-SMX continúa siendo una opción aceptable dado el bajo costo del medicamento y un acceso más fácil con tasas de curación de $74 \%$ y adherencia hasta en $68 \%$ de los pacientes, por lo que en nuestro medio es otra estrategia de manejo tal como se usó con el paciente (18).

Este caso clínico nos muestra la importancia de considerar la PCM aguda/subaguda como un diagnóstico diferencial en pacientes jóvenes con adenopatías y eosinofilia, además buscar nuevas estrategias como la PCR para poder confirmar más rápidamente esta infección y, por último, tener en cuenta al TMP-SMX como una opción en aquellos pacientes con formas leves y dificultades en el acceso al itraconazol.

\section{Referencias}

1. Martinez R. New trends in paracoccidioidomycosis epidemiology. J Fungi. 2017;3(1)

2. Torrado E, Castañeda E, de la Hoz F, Restrepo A. Paracoccidioidomicosis: definición de las áreas endémicas de Colombia. Biomédica. 2000;20:327-34.
3. Restrepo A, Gómez B, Tobón A. Paracoccidioidomycosis: Latin America's Own Fungal Disorder. Curr Fungal Infect Rep. 2012;6:303-11.

4. Shikanai-Yasuda M, Poncio-Mendes R, Lopes-Colombo A, de Queiroz-Telles F, Gonçalves-Kono A, Paniago A, et al. Brazilian guidelines for the clinical management of paracoccidioidomycosis. Rev Soc Bras Med Trop. 2017;50(5):715-40.

5. Zancope-Oliveira R, Vera-Pizzini C, de Medeiros-Muniz M, Francesconi do Valle A, Almeida-Paes R. Diagnostic Aspects of Paracoccidioidomycosis. Curr Trop Med Rep. 2014;1:111-8.

6. Bellissimo-Rodrigues F, Bollela V, Da Fonseca B, Martinez R. Endemic paracoccidioidomycosis: relationship between clinical presentation and patients' demographic features. Med Mycol. 2013;51(3):313-8.

7. Castañeda-Camacho H, Cano-Rosales D. Paracoccidioidomicosis pulmonar Reporte de dos casos. Rev Col Neumol. 2016;28(2):94-100.

8. Sanabria C, Alarcón M, Alarcón I, Jaimes M. Paracoccidioidomicosis - Una enfermedad multisistémica. Acta Med Colomb. 2018;43(2):111-4.

9. Romaneli M, Tardelli N, Tresoldi A, Morcillo A, Pereira R. Acute/subacute paracoccidioidomycosis: A paediatric cohort of 141 patients, exploring clinical characteristics, laboratorial analysis and developing a non-survival predictor. Mycoses. 2019;62(11):999-1005.

10. Ballesteros A, Beltrán S, Patiño J, Bernal C, Orduz R. Paracoccidioidomicosis juvenil diseminada diagnosticada en una niña en área urbana. Biomedica. 2014;34(1):21-8.

11. Mejia-Zuluaga M, Rosas S, Posada-Vélez V, Quintero P. Hypereosinophilia secondary to disseminated paracoccidioidomycosis. Am J Case Rep. 2017;18:1114-7.

12. Reddy S, Yee J, Soi V. False positive anti-hepatitis C (HCV) antibody after a fungal infection. Am J Kidney Dis. 2015;65(4):A71.

13. Scarpelli-Martinelli-Vidal M, Barbaro-Del Negro G, Pardini-Vicentini A, Estivalet-Svidzinski T, Mendes-Giannini M, Fusco-Almeida A, et al. Serological Diagnosis of Paracoccidioidomycosis: High Rate of Inter-laboratorial Variability among Medical Mycology Reference Centers. PLoS Negl Trop Dis. 2014;8(9):e3174.

14. Gaviria M, Rivera V, Muñoz-Cadavid C, Cano L, Williams-Naranjo T. Validation and clinical application of a nested PCR for paracoccidioidomycosis diagnosis in clinical samples from Colombian patients. Braz J Infect Dis. 2015;19(4):376-83.

15. de Souza-Cavalcante R, Sylvestre T, Levorato A, de Carvalho L, Mendes R. Comparison between Itraconazole and Cotrimoxazole in the Treatment of Paracoccidiodomycosis. PLoS Negl Trop Dis. 2014;8(4):e2793.

16. Borges S, da Silva G, Chambela M da C, de Oliveira R de V, Costa R, Wanke B, et al. Itraconazole vs. Trimethoprim-Sulfamethoxazole: A Comparative Cohort Study of 200 Patients With Paracoccidioidomycosis. Med Mycol.2014;52(3):30310

17. Mendes-Peçanha P, de Souza S, Falqueto A, Grão-Veloso T, Ventura-Lírio $\mathbf{L}$, Gonçalves-Ferreira C, et al. Amphotericin B lipid complex in the treatment of severe paracoccidioidomycosis: a case series. Int J Antimicrob Agents. 2016;48(4):30.

18. Nery A, Crepaldi N, Rossi S, Tadano T, Leal-Santos F, Hahn R, et al. Therapeutic response in adult patients with nonsevere chronic paracoccidioidomycosis treated with sulfamethoxazole-trimethoprim: A retrospective study. Am J Trop Med Hyg. 2017;97(2):62. 